\title{
Enhancing the Security of Communication Using Encryption Algorithm Based on ASCII Values of Data
}

\author{
Farheen Qazi ${ }^{1}$, Fozia Hanif Khan ${ }^{2 *}$, Khurram Nawaz Kiani ${ }^{3}$, Shakil Ahmed ${ }^{4}$, \\ Sadiq Ali Khan ${ }^{5}$ \\ ${ }^{1,4}$ Department of Computer Engineering, Sir Syed University of Engineering and \\ Technology Karachi, Pakistan \\ ${ }^{2}$ Department of Mathematics, Sir Syed University of Engineering and Technology \\ Karachi, Pakistan \\ ${ }^{5}$ Department of Computer Science, University of Karachi \\ ${ }^{3}$ Trafix LLC Karachi, Pakistan \\ engr.fq@gmail.com,ms_khans2011@hotmail.com,kkiani@trafix.com, \\ atshakil@yahoo.com,msakhan@uok.edu.pk
}

\begin{abstract}
The best way of exchanging the information is communication, due to this security of communication system become more challenging and typical day by day. Cryptography plays a major role in giving the data security which is transmitted over the internet work. The process of transforming the plain text is called encryption. Plain text is supposed to be the input of every encryption process and the corresponding cipher text is the output, whereas in the process of decryption the situation is opposite. As the process of decryption is converting cipher text in to plane text. This study presents the algorithms based on ASCII values of characteristics in the plane text for the encryption and decryption process. Since this effort has already been done by many researchers, but here we are trying to enhance the security by modifying the previously used string keys for the data encryption and decryption. The algorithm is presented for the symmetric cryptosystem in which same key will be used for the encryption and corresponding decryption process.
\end{abstract}

Keywords: Encryption, decryption, cryptography, 32-bit, 64- bit, transposition, shifted bit, plain text, cipher text, ASCII values.

\section{Introduction}

In today's world we are living with information age and it is almost impossible to see the world without internet. Huge amount of data is being transferred over the internet in every minute between the computers of different users for the purpose of professional and personal reasons. Due to this now it become necessary to secure such data, Physical security is not always a sufficiently feasible solution for such problems due to its cost and efficiency. Therefore the development in the methodology of virtual security become more advance day by day to secure the data. "The word cryptography was come from the "Greek" word "Kryptos" which defined as anything that is hidden obscure, secret or mysterious. Also defined by Yamen Akdeniz, the study of secret writing is cryptography". Cryptography concerned with the different way by which the communication can be prevented from being disclosed through various techniques. There are several ways of doing this procedure so that the only authorized people can read the massage correctly. Cryptography is an art of designing or creating the code or secret massage between sender and receiver. The process encryption is actually tries to make the information hidden or secret as much as possible, encryption is actually the main process of cryptography that 
convert the main text in to some other meaningless and unintelligible information that appears to be randomly generated.

\section{Related Work}

Many encryption algorithms are available in the literature $[1,2,3]$ few of them are incorporate with both substitution and transposition techniques $[4,5]$ and some of them are classified as symmetric key algorithm [6] that contains single key encryption and decryption. Stream cipher or block cipher is usually used by Symmetric-key encryption where as in Asymmetric cryptography [7] pair of key is used for the encryption decryption of massages. Several cryptographic algorithms are given in $[8,9,10]$ which is based on ASC II values of massages to be encrypted.

The advantage of using secret key cryptography is that [16] it is easily to implement, much faster and takes less processing speed as compare to public key cryptography. Secret key cryptography is fast in computation.

Sign et al has proposed [11] data security with secret hiding advance computing, "to increase the diffusion and confusion characteristics of cipher text, Omolara et al [12] has provided a modified Caesar cipher and Vigenere cipher by incorporating techniques from modern ciphers such as xoring key to the first letter of plain text to the second letter". Senthil et al [13] has presented the modern Creaser cipher substitution by using computational intelligence, "Disina et al [14] has built a method of encryption that based on the position of the bit in the message. Purnama et al [15] has used such a method of encryption in which the cipher text generated is legible and make it less suspicious to the cryptanalyst that the text has been encrypted".

This study also deals with ASC II values of massages and providing two algorithms for the modern cryptography the security of the provided algorithm will be more enhanced by the implementation on 16 bits and 32 bits. Experimental results will show the efficiency of these algorithms.

This paper organizes as follows, section 1 gives the introduction with literature reviews, section 2 provides the detail about the decryption procedure, section 3 indicates the steps of encryption and decryption procedures, section 4 shows the provided algorithm's flow chart, section 5 displays the results on 16 and 32 bits, section 6 discusses the results and their efficiency, section 7 concludes the study and in the end there are references.

\section{Proposed Method}

Consider any plaintext $P_{I}$ and let the key be $\kappa$. Now generate the different version of keys by taking the double of first randomly generated value and rest of the keys will be generating by the same procedure. Take any character of the plane text and find its ASC II value, than by adding the $A S C I I$ value in to key value calculate $€ 1$ and by taking the right shift of $€ 1$ calculate $€ 2$. Save the bit value of $€ 2$ to the decryption process and finally get the cipher text $€$ by taking the 2's compliment of the $€ 2$ on 16 bits. The same procedure is applied on 32 bits for more secure encryption procedure.

In the next algorithm we considering the plane text and make it transpose (in the reverse order). Generate the key and calculate the $€ 1$ and $€ 2$ with the same procedure as described above and finally obtained the cipher text with the 2's complement of $€ 2$ on 16 bits. This procedure may also be applied on 32 bits that provides more security as compare to previously generated procedures. 


\subsection{Algorithm based on Two's Complement and Right Shift Method}

\subsubsection{Algorithm for Encryption}

Step 1: Begin

Step 2: Take the plain text message as Input

Step 3: Decide the value of key $\kappa[0]$.

Step 4: Generate different key values $\kappa \mathrm{I}, 1 \leq \mathrm{I} \leq \mathrm{n}$

$\kappa[\mathrm{i}]=\kappa[\mathrm{i}-1]+\kappa[\mathrm{i}-1]$

$\kappa[0]=$ say 25 ;

$\kappa[1]=\kappa[0]+\kappa[0]$;

$\kappa[2]=\kappa[1]+\kappa[1] ;$ Etc $\ldots$

Step 5: Add the resultant Key in the value of plain text.

Step 6: take the Right shift of the output of step 5 once.

Step 7: Take the 2's Complement of the output of step 6

Step 8: Pass on the Cipher text

\subsubsection{Decryption Algorithm}

Step 1: Start

Step 2: Taking 2's Complement of Cipher text.

Step 3: Left shift the output of step 2 once.

Step 4: Add the shifted bit in output of step 3 .

Step 5: Subtract the key values from the resultant of step 4.

Step 6: obtain the ASCII values from step 5.

Step 7: Convert $A S C I I$ values to plain text.

\subsection{For 16-bits}

3.2.1 Encryption

\begin{tabular}{cccccccc} 
S. & $\mathbf{P . T}$ & $\boldsymbol{A S C I I} \cdot \boldsymbol{V}$ & $\boldsymbol{\kappa}$ & $\boldsymbol{\epsilon 1}$ & $\boldsymbol{\epsilon 2}$ & S.Bit & $\boldsymbol{\epsilon}$ \\
$\mathbf{N o .}$ & & & & & & & \\
$\mathbf{1}$ & $\mathrm{H}$ & 72 & 25 & 97 & 48 & 1 & 65488 \\
$\mathbf{2}$ & $\mathrm{I}$ & 73 & 50 & 123 & 61 & 1 & 65475 \\
$\mathbf{3}$ & $\mathrm{D}$ & 68 & 100 & 168 & 84 & 0 & 65452 \\
$\mathbf{4}$ & $\mathrm{E}$ & 69 & 200 & 269 & 134 & 1 & 65402 \\
\hline
\end{tabular}

P.T = Plain text,

ASCII.V = ASCII Value,

$\kappa=$ Key Value,

$€ 1=$ ASCII. $V+\kappa$,

$€ 2=$ Right Shifted $\mathrm{C} 1$ once,

$€=$ Ciphertext (2's Complement of $€ 2$ on 16-bits).

\subsubsection{Decryption}

\begin{tabular}{cccccc} 
S. & $\boldsymbol{\epsilon}$ & $\mathbf{P 3}$ & $\mathbf{P 2}$ & $\mathbf{P 1}$ & P.T \\
No. & & & & & \\
$\mathbf{1}$ & 65488 & 48 & 97 & 72 & H \\
$\mathbf{2}$ & 65475 & 61 & 123 & 73 & I \\
$\mathbf{3}$ & 65452 & 84 & 168 & 68 & D \\
$\mathbf{4}$ & 65402 & 134 & 269 & 69 & E \\
\hline
\end{tabular}


$€=$ ASCII equivalent of cipher text,

Р3 $=2$ 's Complement of $€$,

Р2 $=$ Left shifted P3 once + S.Bit,

$\mathrm{P} 1=\mathrm{P} 2-\kappa$

P.T $=$ Real Plain text Characters.

\subsection{For 32-bits}

\subsubsection{Encryption}

\begin{tabular}{cccccccc} 
S. & F.T & $\boldsymbol{A S C I I} . \boldsymbol{V}$ & $\boldsymbol{\kappa}$ & $\boldsymbol{\epsilon 1}$ & $\boldsymbol{\epsilon 2}$ & S.Bit & $\boldsymbol{\epsilon}$ \\
No. & & & & & & & \\
$\mathbf{1}$ & H & 72 & 25 & 97 & 48 & 1 & 4294967248 \\
$\mathbf{2}$ & $\mathrm{I}$ & 73 & 50 & 123 & 61 & 1 & 4294967235 \\
$\mathbf{3}$ & $\mathrm{D}$ & 68 & 100 & 168 & 84 & 0 & 4294967212 \\
$\mathbf{4}$ & $\mathrm{E}$ & 69 & 200 & 269 & 134 & 1 & 4294967162 \\
\hline
\end{tabular}

P.T = Plain text,

ASCII.V = ASCII Value,

$\kappa=$ Key Value,

$€ 1=A S C I I . V+\kappa$,

$€ 2=$ Right Shifted $\mathrm{C} 1$ once,

$€=$ Cipher text (2's Complement of C2 on 32-bits).

\subsubsection{Decryption}

\begin{tabular}{rccccc} 
S. & $\boldsymbol{E}$ & $\mathbf{P 3}$ & $\mathbf{P 2}$ & P1 & 疋.T \\
No. & & & & & \\
$\mathbf{1}$ & 4294967248 & 48 & 97 & 72 & $\mathrm{H}$ \\
$\mathbf{2}$ & 4294967235 & 61 & 123 & 73 & $\mathrm{I}$ \\
$\mathbf{3}$ & 4294967212 & 84 & 168 & 68 & $\mathrm{D}$ \\
$\mathbf{4}$ & 4294967162 & 134 & 269 & 69 & $\mathrm{E}$ \\
\hline
\end{tabular}

$€=$ ASCII equivalent of ciphertext,

Р3 $=2$ 's Complement of $€$,

Р2 $=$ Left shifted P3 once + S.Bit,

$\mathrm{P} 1=\mathrm{P} 2-\kappa$,

P.T $=$ Real Plaintext Characters. 


\subsection{Flow Chart for Encryption \& Decryption}

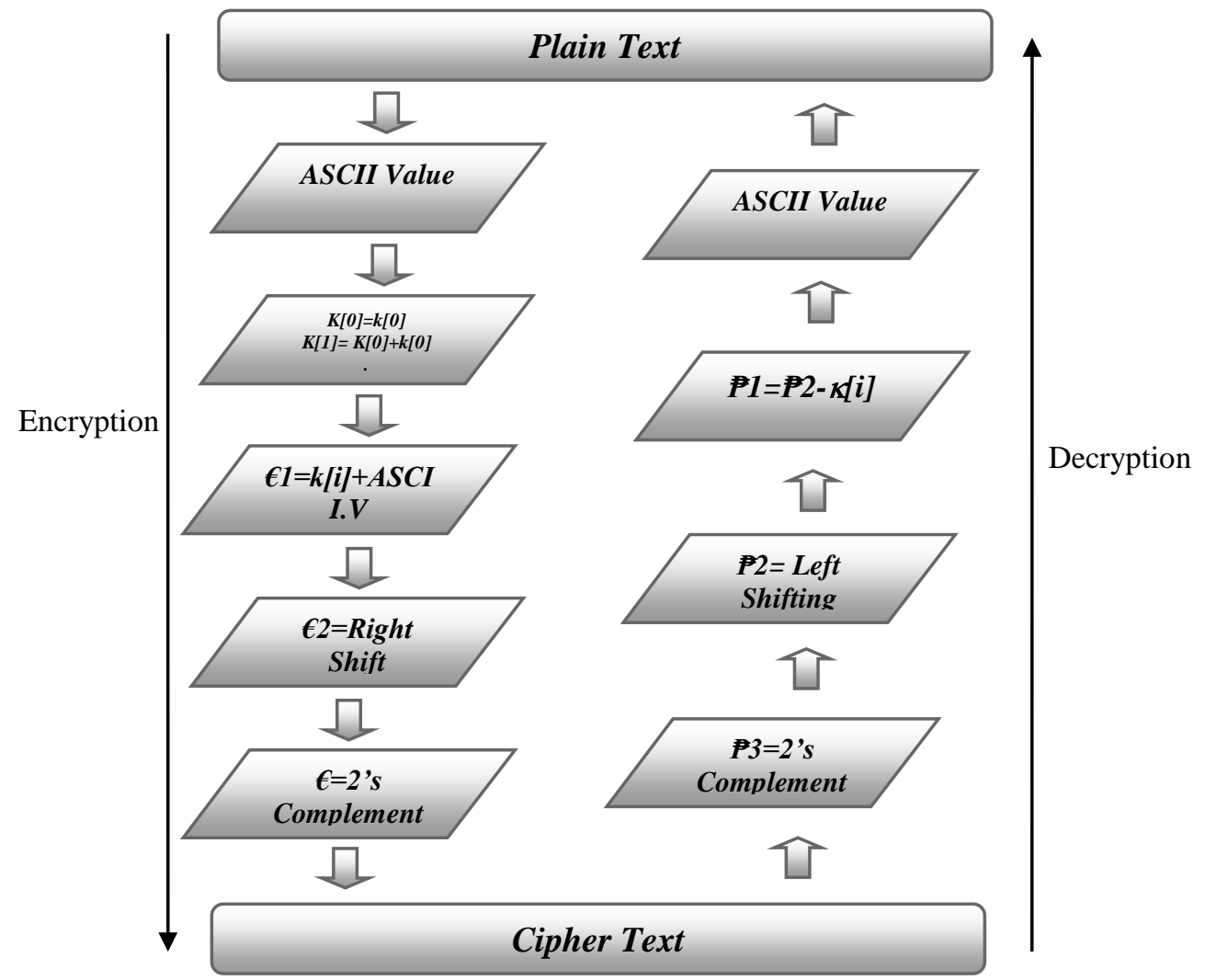

\subsection{Algorithm based on Two's Complement, Transposition and Right Shift Method}

\subsubsection{Algorithm for Encryption}

Step 1: Begin

Step 2: Take the plain text as input.

Step 3: Applying transposition on resultant of step 2

Step 4: Decide the value of key $\kappa[0]$.

Step 5: Generate different key values $\kappa \mathrm{I}, 1 \leq \mathrm{I} \leq \mathrm{n}$

$\mathrm{K}[\mathrm{i}]=\kappa[\mathrm{i}-1]+\kappa[\mathrm{i}-1]$;

$\kappa[0]=$ say 25 ;

$\kappa[1]=\kappa[0]+\kappa[0]$;

$\kappa[2]=\kappa[1]+\kappa[1] ;$ Etc...

Step 6: Add resultant Key in the value of plain text.

Step 7: Right shift the output of step 6 once

Step 8: Taking 2's Complement the output of step 7

Step 9: Pass on the Cipher text

\subsubsection{Decryption Algorithm}

Step 1: Begin

Step 2: Taking 2's Complement of Cipher text.

Step 3: Left shift the output of step 2 once.

Step 4: Add the shifted bit in output of step 3.

Step 5: Subtract the key values from the resultant of step 4.

Step 6: Obtain the ASCII values from step 5. 
Step 7: Perform back transposition on output of step 6.

Step 8: Convert ASCII values to plain text.

\subsection{For 16-bits}

\subsubsection{Encryption}

\begin{tabular}{ccccccccc} 
S. & 尹.T & Trans & $\boldsymbol{A S C I I . ~} \boldsymbol{V}$ & $\boldsymbol{\kappa}$ & $\boldsymbol{\epsilon 1}$ & $\boldsymbol{\epsilon 2}$ & S.Bit & $\boldsymbol{\epsilon}$ \\
No. & & & & & & & & \\
$\mathbf{1}$ & H & E & 69 & 25 & 94 & 47 & 0 & 65489 \\
$\mathbf{2}$ & I & D & 68 & 50 & 118 & 59 & 0 & 65477 \\
$\mathbf{3}$ & D & I & 73 & 100 & 173 & 86 & 1 & 65450 \\
$\mathbf{4}$ & E & H & 72 & 200 & 272 & 136 & 0 & 65400 \\
\hline
\end{tabular}

P.T = Plain text,

Trans $=$ Transposition (in reverse order),

ASCII.V = ASCII Value,

$\kappa=$ Key Value,

$€ 1=A S C I I . V+\kappa$,

$€ 2=$ Right Shifted $€ 1$ once,

$€=$ Cipher text (2's Complement of $€ 2$ on 16-bits).

\subsubsection{Decryption}

\begin{tabular}{|c|c|c|c|c|c|c|}
\hline $\begin{array}{l}\text { S. } \\
\text { No. }\end{array}$ & $€$ & $\mathbf{P 3}$ & $\mathbf{P 2}$ & $\mathbf{P 1}$ & B.Trans & P.T \\
\hline 1 & 65489 & 47 & 94 & 69 & $\mathrm{E}$ & $\mathrm{H}$ \\
\hline 2 & 65477 & 59 & 118 & 68 & D & I \\
\hline 3 & 65450 & 86 & 173 & 73 & I & D \\
\hline 4 & 65400 & 136 & 272 & 72 & $\mathrm{H}$ & $\mathrm{E}$ \\
\hline
\end{tabular}

$€=A S C I I$ equivalent of cipher text,

P3 $=2$ 's Complement of $€$,

Р2 $=$ Left shifted P3 once + S.Bit,

$\mathrm{P} 1=\mathrm{P} 2-\kappa$,

B. Trans $=$ Back Transposition,

$\mathrm{P}=$ Real Plain text Characters.

\subsection{For 32-bits}

\subsubsection{Encryption}

\begin{tabular}{|c|c|c|c|c|c|c|c|c|}
\hline $\begin{array}{c}\text { S. } \\
\text { No. }\end{array}$ & P.T & Trans & $\begin{array}{c}A S C I I . \\
V\end{array}$ & $\kappa$ & $€ 1$ & $€ 2$ & S.Bit & $€$ \\
\hline 1 & $\mathrm{H}$ & $\mathrm{E}$ & 69 & 25 & 94 & 47 & 0 & 4294967249 \\
\hline 2 & I & D & 68 & 50 & 118 & 59 & 0 & 4294967237 \\
\hline 3 & $\mathrm{D}$ & I & 73 & 100 & 173 & 86 & 1 & 4294967210 \\
\hline 4 & $\mathrm{E}$ & $\mathrm{H}$ & 72 & 200 & 272 & 136 & 0 & 4294967160 \\
\hline
\end{tabular}

P.T = Plain text,

Trans = Transposition (in reverse order),

ASCII.V = ASCII Value,

$\kappa=$ Key Value, 
$€ 1=$ ASCII.V $+\kappa$,

$€ 2=$ Right Shifted $\mathrm{C} 1$ once,

$€=$ Cipher text (2's Complement of $€ 2$ on 32-bits).

\subsubsection{Decryption}

\begin{tabular}{ccccccc}
$\mathbf{S .}$ & $\boldsymbol{E}$ & $\mathbf{P 3}$ & $\mathbf{P 2}$ & $\mathbf{P 1}$ & B.Trans & P.T \\
No. & & & & & & \\
$\mathbf{1}$ & 4294967249 & 47 & 94 & 69 & $\mathrm{E}$ & $\mathrm{H}$ \\
$\mathbf{2}$ & 4294967237 & 59 & 118 & 68 & $\mathrm{D}$ & $\mathrm{I}$ \\
$\mathbf{3}$ & 4294967210 & 86 & 173 & 73 & $\mathrm{I}$ & $\mathrm{D}$ \\
$\mathbf{4}$ & 4294967160 & 136 & 272 & 72 & $\mathrm{H}$ & $\mathrm{E}$ \\
\hline
\end{tabular}

$€=A S C I I$ equivalent of cipher text,

P3 $=2$ 's Complement of $€$,

$\mathrm{P} 2=$ Left shifted P3 once + S.Bit,

$\mathrm{P} 1=\mathrm{P} 2-\kappa$,

B. Trans $=$ Back Transposition,

P.T $=$ Real Plain text Characters.

\section{Results and Discussion}

In the experiments we have calculated the executing time of the proposed algorithms on 16 and 32 bits using MATLAB simulations. The comparisons have been made with the two previously methodologies [5] and [8] in which the execution time has been evaluated on 4 bits. The results given by table 1 and table 2 are showing very good performance of the proposed algorithm based on two's complement and transposition method for 16 and 32 bits as it is very much less as compare to the technique 1 [8] which is on 4 bits, where as it is almost same as compare to technique 2 [5] except for some places. But in comparison with 4 bits they are much promising results for 16 bits as far as the security of cryptography is concerned. Similarly for the Transposition and Right Shift Method same observations can be seen by table 3 and table 4 for 16 and 32 bits respectively. The execution time is very less as compare to technique 1 given by [8]. The comparison made by proposed algorithm for 32 bits with the technique 2 given by [5] which is on 4 bits, is showing very less difference in the execution time.

Table 1. Comparison between Previously Developed Methodologies and Two's Complement Transposition Method for 16-bits

\begin{tabular}{|c|c|c|c|}
\hline \multirow{2}{*}{$\begin{array}{l}\text { Size of } \\
\text { Plaintext }\end{array}$} & \multicolumn{3}{|c|}{ Encryption \& Decryption Execution Tlime (ms) } \\
\hline & $\begin{array}{l}\text { Execution time for } \\
\text { existing algorithm (4- } \\
\text { bit) in } \\
\text { ms }\end{array}$ & $\begin{array}{l}\text { Execution time for } \\
\text { existing algorithm (4- } \\
\text { bit) in } \\
\text { ms }\end{array}$ & $\begin{array}{c}\text { Execution time for } \\
\text { proposed algorithm } \\
\text { (16-bit) in } \\
\mathrm{ms}\end{array}$ \\
\hline 2 & 322 & 15 & 15 \\
\hline 4 & 3679 & 15 & 16 \\
\hline 6 & 3861 & 16 & 26 \\
\hline 8 & 4748 & 16 & 37 \\
\hline 10 & 5543 & 30 & 52 \\
\hline
\end{tabular}


Table 2. Comparison between Previously Developed Methodologies and Two's Complement Transposition Method for 32-bit

\begin{tabular}{|c|c|c|c|}
\hline \multirow{2}{*}{$\begin{array}{c}\text { Size of } \\
\text { Plaintext }\end{array}$} & \multicolumn{3}{|c|}{ Encryption \& Decryption Execution Time (ms) } \\
\cline { 2 - 4 } & $\begin{array}{c}\text { Execution time for } \\
\text { existing algorithm } \\
\text { (4-bit) in } \\
\text { ms }\end{array}$ & $\begin{array}{c}\text { Execution time for } \\
\text { existing algorithm } \\
\text { (4-bit) in } \\
\text { ms }\end{array}$ & $\begin{array}{c}\text { Execution time for } \\
\text { proposed algorithm } \\
\text { (32-bit) in } \\
\text { ms }\end{array}$ \\
\hline 2 & 322 & 15 & 31 \\
\hline 4 & 3679 & 15 & 33 \\
\hline 6 & 3861 & 16 & 42 \\
\hline 8 & 4748 & 16 & 67 \\
\hline 10 & 5543 & 30 & 98 \\
\hline
\end{tabular}

Table 3. Comparison between Previously Developed Methodologies and Transposition and Right Shift Method for 16-bit

\begin{tabular}{|c|c|c|c|}
\hline \multirow{2}{*}{$\begin{array}{c}\text { Sive of } \\
\text { Plaintext }\end{array}$} & \multicolumn{3}{|c|}{ Encryption \& Decryption Execution Time (ms) } \\
\cline { 2 - 4 } & $\begin{array}{c}\text { Execution time for } \\
\text { existing algorithm (4- } \\
\text { bit) in } \\
\text { ms }\end{array}$ & $\begin{array}{c}\text { Execution time for } \\
\text { existing algorithm (4- } \\
\text { bit) in } \\
\text { ms }\end{array}$ & $\begin{array}{c}\text { Execution time for } \\
\text { proposed algorithm (16- } \\
\text { bit) in } \\
\text { ms }\end{array}$ \\
\hline 2 & 322 & 15 & 18 \\
\hline 4 & 3679 & 15 & 19 \\
\hline 6 & 3861 & 16 & 28 \\
\hline 8 & 4748 & 16 & 50 \\
\hline 10 & 5543 & 30 & 62 \\
\hline
\end{tabular}

Table 4. Comparison between Previously Developed Methodologies and Transposition and Right Shift Method for 32-bit

\begin{tabular}{|c|c|c|c|}
\hline \multirow{2}{*}{$\begin{array}{c}\text { Sire of } \\
\text { Plaintext }\end{array}$} & \multicolumn{3}{|c|}{ Encryption \& Decryption Execution Time (ms) } \\
\hline & $\begin{array}{l}\text { Execution time for } \\
\text { existing algorithm (4- } \\
\text { bit) in } \\
\text { ms }\end{array}$ & $\begin{array}{l}\text { Execution time for } \\
\text { existing algorithm (4- } \\
\text { bit) in } \\
\text { ms }\end{array}$ & $\begin{array}{l}\text { Execution time for } \\
\text { proposed algorithm (32- } \\
\text { bit) in } \\
\text { ms }\end{array}$ \\
\hline 2 & 322 & 15 & 40 \\
\hline 4 & 3679 & 15 & 52 \\
\hline 6 & 3861 & 16 & 99 \\
\hline 8 & 4748 & 16 & 125 \\
\hline 10 & 5543 & 30 & 171 \\
\hline
\end{tabular}

\section{Conclusion}

The above study have presented two algorithm based on ASC II values of the data to enhance the security of communication regarding to cryptosystem. The proposed idea has been made possible by using the transposition and two's complement of the input values. The implementations have been shown on 16 and 32 bits of both the algorithms and experimental results have given the execution time of the related study. The study concludes that the proposed idea is not only good contribution in the literature of 
cryptography but also provides more security in the text cryptography as far as the security is concerned. The provided algorithm is fast and reliable when compared with the other algorithms.

\section{Future Scope}

We can apply different schemes of Classical cipher to develop algorithms to enhance the security of communication regarding cryptosystem. Also we can develop algorithm not only for 16 or 32 bit but for $64,128,254$ or higher bits for secure communication.

\section{References}

[1] Z. Yunpeng, Z. Yu, W. Zhong and R. O. Sinnott "Index-Based Symmetric DNA Encryption Algorithm", $20114^{\text {th }}$ International Congress on Image and Signal Processing, pp. 2290 2294,978-1- 4244-93067/11/\$26.00 @2011 IEEE.

[2] G. Singh, A. K Singla and K.S. Sandha, "Throughput Analysis of Various Encryption Algorithms", International Journal of Computer Science and Technology, Vol. 2, Issue 3, September 2011.

[3] D.S.A. Elminaam, H.M.A. Kader and M. M. Hadhoud, "Evaluating the Performance of Symmetric Encryption Algorithms", International Journal of Network Security, Vol.10, No.3, PP.216-222, May 2010.

[4] Dr. A.P.A.G. Deshmukh, Dr. R. Qureshi, "Transparent Data Encryption- Solution for Security of Database Contents", (IJACSA) International Journal of Advanced Computer Science and Applications, Vol. 2, No.3, March 2011"

[5] Satyajeet R. Shinge et al, An Encryption Algorithm Based on ASCII Value of Data (IJCSIT) International Journal of Computer Science and Information Technologies, Vol. 5 (6) , 2014, 7232-7234.

[6] Gurjeevan Singh, Ashwani Kumar Singla, K.S. Sandha, "Throughput Analysis of Various Encryption Algorithms", International Journal of Computer Science and Technology, Vol. 2, Issue 3, Septemver 2011.

[7] Diaa Salama Abd Elminaam, Hatem Mohamed Abdual Kader, and Mohiy Mohamed Hadhoud, "Evaluating the Performance of Symmetric Encryption Algorithms", International Journal of Network Security, Vol.10, No.3, PP.216-222, May 2010.

[8] A. Mathur, "A Research paper: An ASCII value based data encryption algorithm and its comparison with other symmetric data encryption algorithms", International Journal on Computer Science and Engineering (IJCSE), Vol. 4, pp. 1650-1657, Sep 2012 ISSN: 0975- 3397.

[9] U. Singh, U. Garg, "An ASCII value based text data encryption System", International Journal of Scientific and Research Publications, Volume 3, pp.1-5, November 20131 ISSN 2250-3153.

[10] M.P. Uddin, A. Marjan and M.R. Islam , "Developing a cryptographic algorithm based on ASCII conversions and a cyclic mathematical function", Informatics, Electronics \& Vision (ICIEV), 2014, International Conference .

[11] A. Singh , U. Jauhari, "Data Security by Preprocessing the Text with Secret Hiding", Advanced Computing: An International Journal (ACIJ), Vol.3, No.3, May 2012".

[12] Omolara, O. E., A. I. Oludare, and S. E. Abdulahi. "Developing a Modified Hybrid Caesar Cipher and Vigenere Cipher for Secure Data Communication." Computer Engineering and Intelligent Systems 5.5 (2014): 34-46

[13] Senthil, K., K. Prasanthi, and R. Rajaram. "A modern avatar of Julius Caesar and Vigenere cipher." Computational Intelligence and Computing Research (ICCIC), 2013 IEEE International Conference on. IEEE, 2013.

[14] Disina, Abdulkadir Hassan. "Robust Caesar Cipher against frequency cryptanalysis using bi-directional shifting." Diss. Universiti Tun Hussein Onn Malaysia, 2014.

[15] Purnama, Benni, and AH Hetty Rohayani. "A New Modified Caesar Cipher Cryptography Method with Legible Ciphertext from a Message to be Encrypted.” Procedia Computer Science 59 (2015): 195-204.

[16] Delfs, Hans, and Helmut Knebl. "Public-Key Cryptography." Introduction to Cryptography". Springer Berlin Heidelberg, 2015. 49-106. 


\section{Authors}
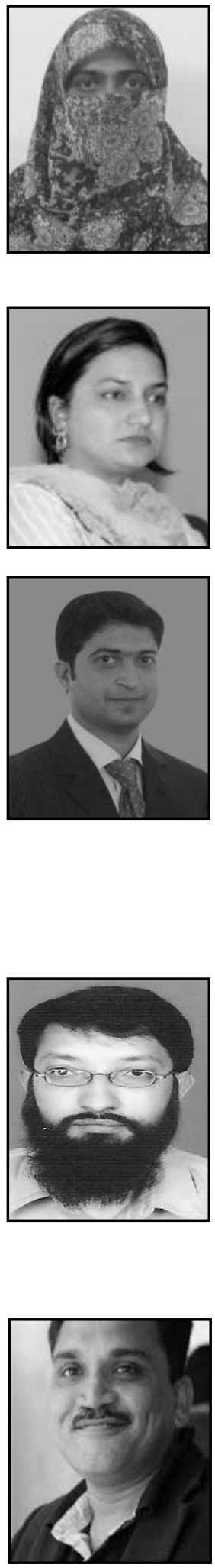

Farheen Qazi is a $\mathrm{Ph}$. D Scholar. She obtained her BS in Computer Engineering and MS in Computer Engineering (Specialization in Computer Networks) from Sir Syed University of Engineering and Technology, Karachi, Pakistan. Since 2008 she is working as a Lecturer in Department of Computer Engineering of Sir Syed University of Engineering and Technology, Karachi, Pakistan. Her research interests are Cryptography, Network Security, Artificial Intelligence and Wireless Sensor Network.

Fozia Hanif khan working as an Associate Professor in the Department of Mathematics of Sir Syed University of Engineering and technology, Karachi, Pakistan. She has done her Ph. D from University of Karachi University in Operations Research. Her fields of interest are cryptography, graph theory, Optimization Network Security and Wireless sensors Networks

Khurram Nawaz Kiani serves as Associate Project Manager at TRAFiX Karachi office. He has extensive knowledge of all phases of the Software Development Life Cycle (SDLC). Mr. Kiani has broad based IT expertise, excellent analysis and design skills and in depth knowledge of industrial and financial application development. He has extensive hands on experience in designing and developing of distributed, network and web-based applications using Microsoft and other technologies. He obtained his BS in Computer Engineering from Sir Syed University of Engineering and Technology, Karachi, Pakistan. His fields of interest are web based distributed applications and mobile development.

Shakil Ahmed is currently serving as Chairman, Department of Computer and Software Engineering, Sir Syed University of Engineering and Technology Karachi, Pakistan. He completed his $\mathrm{PhD}$ from University Putra Malaysia in 2014. He did his Master's from NED University of Engineering and Technology, Karachi Pakistan in 2006. He has around 20 national and international publications in Journals and Conferences. His primary area of research is Information Security. Other research areas include Data Mining, Information Theory.

M.Sadiq Ali Khan received his Ph.D Degree from KU in 2011 and his BS \& MS Degree in Computer Engineering from SSUET in 1998 and 2003 respectively. Since 2003 he is serving Computer Science Department, University of Karachi as an Assistant Professor. He has about 18 years of teaching and research experience and his research areas includes Data Communication \& Networks, Network Security, Cryptography issues and Security in Wireless Networks. He is the member of CSI, PEC, IEEE and NSP. He is currently Wise Chair IEEE Computer Society Karachi Section. 